\title{
Characteristics of Tsunami Damage to the Tide Walls in Iwate Prefecture from the Great East Japan Earthquake
}

\author{
Tetsuya Oyamada, Shunsuke Hanehara, Hideaki Deto and Shoji Iwasaki \\ Department of Civil and Environmental Engineering, Iwate University, Iwate 0208551, Japan
}

\begin{abstract}
This research paper describes the tsunami damage to tide walls observed using aerial photographs and field investigations at 13 locations along the coast of Iwate Prefecture, where significant tsunami damage occurred as a result of the Great East Japan Earthquake. This paper also summarizes the characteristics of the damage. The tide walls in Iwate Prefecture were constructed on a ria (hilly type) coast, this geographical feature of Iwate being different from that of the other disaster areas, primarily Miyagi and Fukushima Prefectures. The results of our investigations show that all the devastated tide walls suffered from overflow before being damaged. In particular, the sloping and vertical type tide walls with slope protection were damaged by scouring of the infill sand or back fill soil due to the overflow of the tsunami. In the case of vertical type tide walls without slope protection, damage was caused by scouring of the back fill soil.
\end{abstract}

Key words: Tide wall, Great East Japan Earthquake, tsunami.

\section{Introduction}

The Great East Japan Earthquake occurred at 2:46 p.m. on March 11, 2011 at a depth of $24 \mathrm{~km}$ off the coast of Sanriku [1]. Registering a magnitude of 9.0 on the Richter scale, it was the largest earthquake in recorded Japanese history.

The magnitude of the earthquake itself and the damage it caused were tremendous, but investigations after it occurred reveal that most of the human suffering and physical damage were due to the tsunami that the earthquake triggered, in particular, the tsunami damage to the Pacific coastline was enormous. According to a report released by the Development Bank of Japan, the catastrophic damage to the coastal structures and their surroundings in the three Tohoku area prefectures, Iwate, Miyagi and Fukushima, amounted to 10 trillion yen in total [2]. The tsunami hit the areas in different ways. The extensive movement of the earth's crust was a significant factor but the differences in coastal

Corresponding author: Tetsuya Oyamada, Dr., associate professor, research field: concrete material. E-mail: oyamada@iwate-u.ac.jp. topography also had an important influence [3]. Miyagi and Fukushima Prefectures have comparatively even coastlines and wide coastal plains extending significantly inland. In contrast, Iwate Prefecture has an indented coastline known as a ria coast. In the Design Manual for Coastal Facilities, 2000 [4], the former coastline is classified as a plain type and the latter as a hilly type. In implementing safety measures against future tsunami disasters, it is essential to take account of the types of damage that occurred. To this end, we carried out a preliminary investigation of those tide walls which underwent particularly severe damage and have summarized our findings in this research paper.

\section{Outline of Investigation}

Table 1 lists the tide walls that were investigated, together with their structural types. The classifications of tide walls in the Design Manual for Coastal Facilities 2000, referred to this paper in Fig. 1. We visited 13 locations to investigate tide walls with various kinds of back slope protection. The locations of the tide walls investigated are shown in Fig. 2. Almost 
Table 1 Tide walls investigated and their structural types.

\begin{tabular}{lllll}
\hline No. & Investigated Area & Municipality & Type of tide wall & Type of back slope protection \\
\hline 1 & Tofugaura Coast & Noda Village & Sloping & Concrete block \\
2 & Taro Fishery Port Coast & Miyako City & Sloping & Concrete block \\
3 & Yamada Fishery Port Coast & Yamada Town & Vertical & None \\
4 & Koyadori Coast & Yamada Town & Vertical & Concrete block \\
5 & Funakoshi Fishery Port Coast & Yamada Town & Vertical & Concrete block \\
6 & Otsuchi River & Otsuchi Town & Sloping & Concrete block \\
7 & Kojirahama Fishery Port Coast & Kamaishi City & Vertical & None \\
8 & Yoshihama Agricultural Field Coast & Ofunato City & Vertical & Concrete block \\
9 & Kadonohama Fishery Port & Rikuzentakata City & Vertical & None \\
10 & Mutsugahama Coast & Rikuzentakata City & Sloping & Concrete block \\
11 & Otomo Agricultural Field Coast & Rikuzentakata City & Sloping & Asphalt pavement \\
12 & Takada Coast & Rikuzentakata City & Sloping & Concrete block \\
13 & Kesen River & Rikuzentakata City & Sloping & Concrete block \\
\hline
\end{tabular}

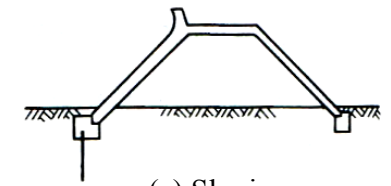

(a) Sloping
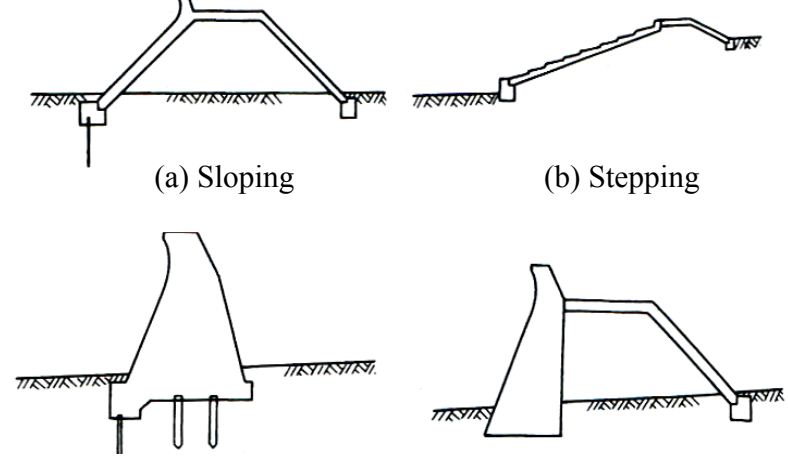

(c) Vertical

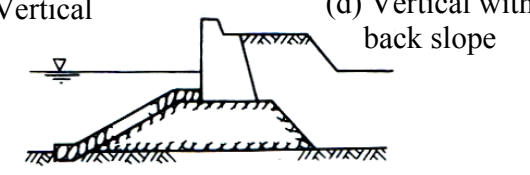

(e) Mixed

Fig. 1 Types of tide walls: (a) sloping; (b) stepping; (c) vertical; (d) vertical with back slope; (e) mixed.

all of the coastal areas in Iwate Prefecture were struck by the tsunami, but the extensive damage occurred mostly in the southern coastal area. Several factors were involved in the damage to coastal structures, including the height of the tsunami, types of foundation structures and installed environments. Although all these factors need to be assessed, we focus on the situation regarding damage to the coastal structures in this study.

In some places, the local situation prevented us from investigation. For example, the damage in some places was so great that the locations of tide walls could not be confirmed. In other places, urgent restoration work prevented us from observing the damage situation. In anticipation of these circumstances, we used Google Earth to compare the situation before and after the tsunami in preparation for the site visits to visually observe the damage.

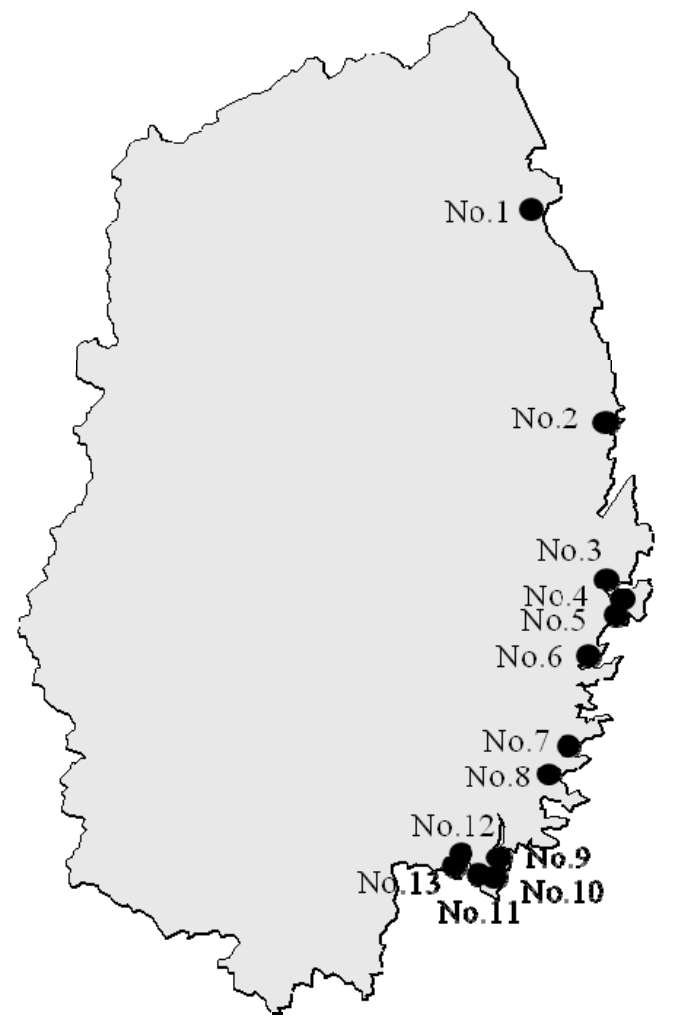

Fig. 2 Locations of researched tide walls. 


\section{Results of Investigation}

The tide walls investigated on the coast of Iwate Prefecture are listed in Table 1 are classified into three main categories with respect to structural type and back slope protection method, as shown in Table 2.

The sloping tide wall is commonly used for river banks and on sandy beaches, and the vertical type tide wall without back slope protection is typically used for fishery ports. Our investigation found that these types yielded characteristic differences with respect to damage, as described in detail below. All the tide walls that underwent damage suffered from overflow by the tsunami.

\subsection{Sloping Tide Wall}

Fig. 3 shows an aerial photograph of the severely damaged Tofugaura Coast area (No. 1). The slightly inclined vertical white line falling a little to the right of the photograph's center indicates that a concrete foundation and concrete superstructures are scattered on both the sea and land sides. A section of the damaged tide wall is shown in Fig. 4. There is sea sand inside the wall's structure. Fig. 5 shows the collapsed tide wall. In this area, the sea side slope, or the front side slope, of the tide wall has collapsed toward the sea, the concrete blocks which protected the back slope have fallen towards the land side, and the infill sand has flowed out. This damage suggests that spilling waves first knocked down the concrete blocks and scoured the infill sand, and the backrush then caused the front slope to fall down.

Fig. 6 shows an aerial photograph of the coast at Taro Fishery Port (No. 2). In this area, two tide walls intersect at their centers to form an $X$ shape. The tsunami overflowed both of them and damaged the shore side tide walls. The southern parts of the tide walls had the surface of the back slopes torn off and the infill sand washed out, as shown in Fig. 7. In contrast, their northern parts suffered from more devastating damage. Fig. 8 shows the damage to the northern part photographed from the sea side. The
Table 2 Classification of investigated tide walls.

\begin{tabular}{lll}
\hline Structural type & \multicolumn{3}{l}{ Back slope protection } & No. \\
\hline Sloping & Provided & $1,2,6,10,11,12,13$ \\
Vertical & Provided & $1,4,5,8$ \\
& Not provided & $3,7,9$ \\
\hline
\end{tabular}

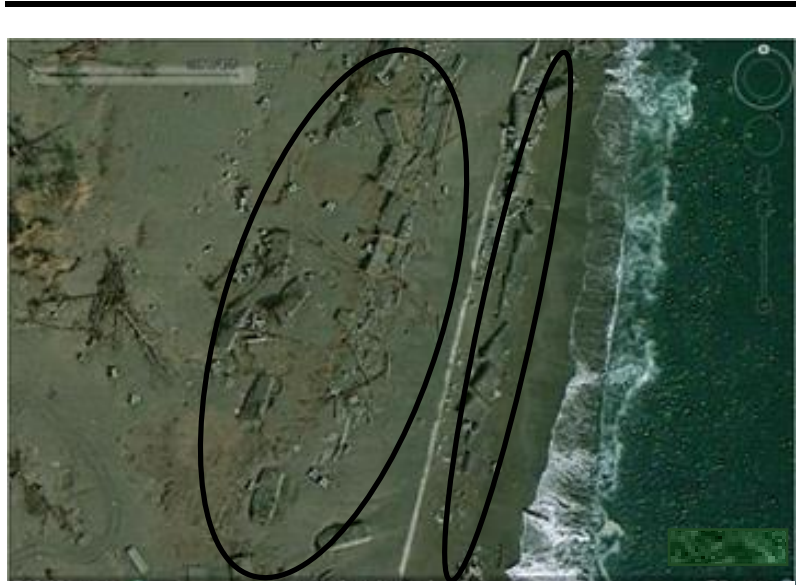

Fig. 3 Aerial photograph of Tofugaura Coast (No. 1).

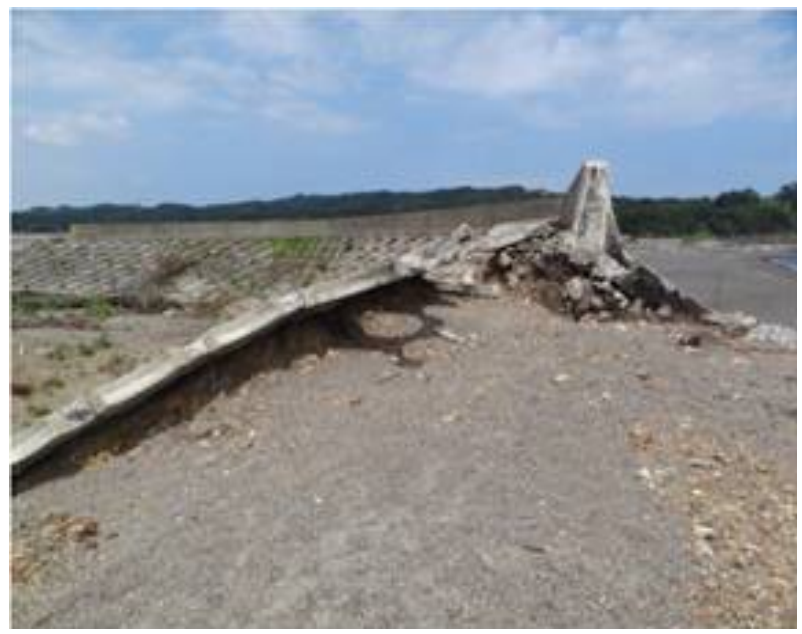

Fig. 4 Section of tide wall (No. 1).

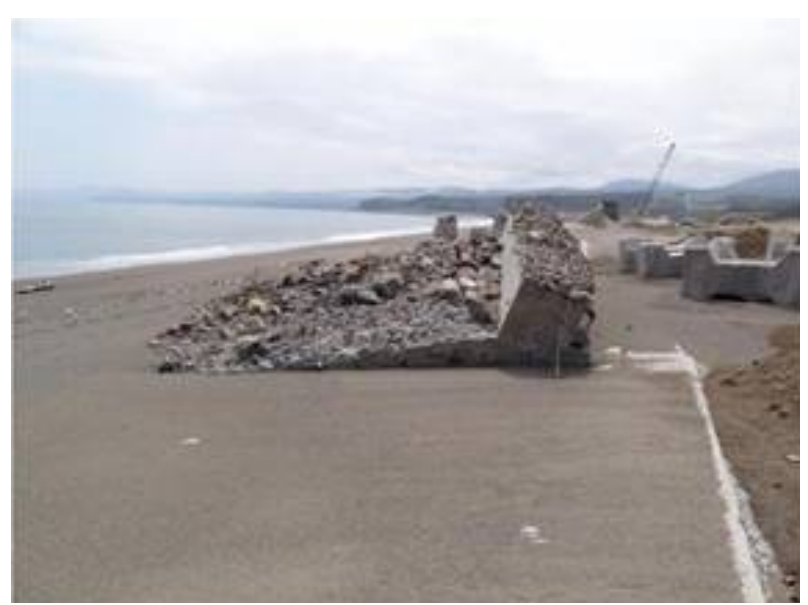

Fig. 5 Collapsed tide wall (No. 1). 


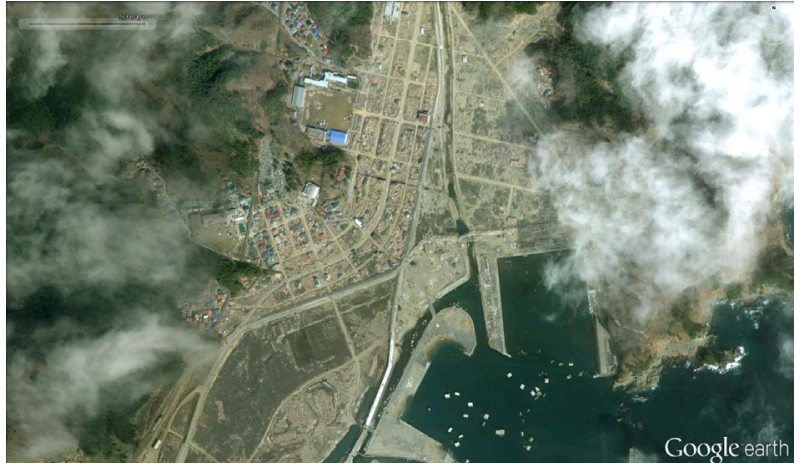

Fig. 6 Aerial photograph of coast at taro fishery port (No. 2).

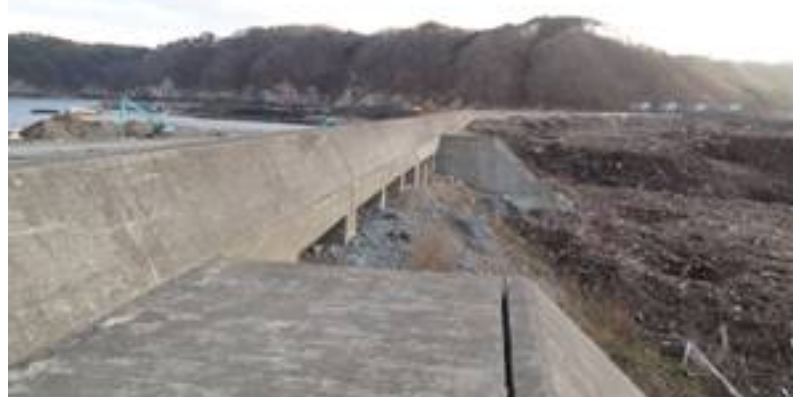

Fig. 7 Damaged tide wall (south side) (No. 2).

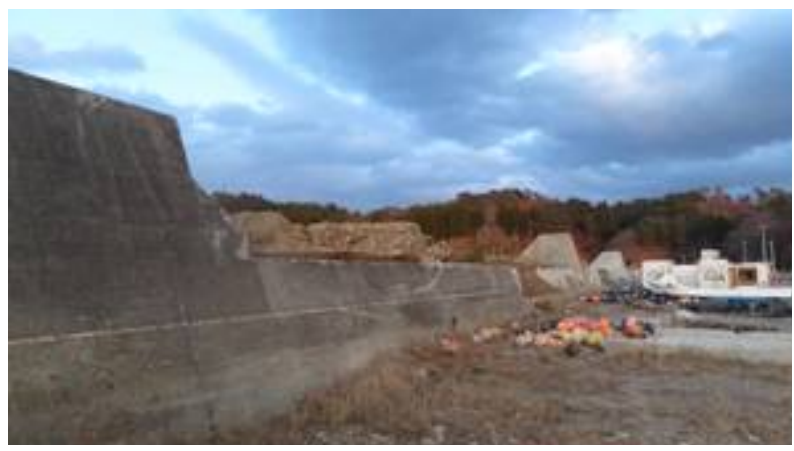

Fig. 8 Damaged tide wall (north side) (No. 2).

concrete blocks of the front side slope have been knocked down and the infill sand washed out. Only the concrete which supported the front slope remains.

Fig. 9 shows an aerial photograph of the Otsuchi River (No. 6) bank after damage by the tsunami. It can be seen that the river bank has been broken and the rear side of the bank flooded with overflowed water. The back slope of the damaged river bank is shown in Fig. 10. Similar to the other cases described above, the concrete blocks of the river banks have slipped off, as shown in Fig. 11, and pools of water can be identified.

Based on our investigations, it was judged that - to a greater or lesser extent-the sloping tide walls in other areas suffered similar damage.

As observed above, a characteristic of the damage to the sloping tide walls is believed to be the scouring of infill sand due to overflow by the tsunami, which in turn caused the structure to collapse.

\subsection{Vertical Tide Wall with Back Slope Protection}

Fig. 12 shows an aerial photograph of the coast at Yoshihama Agricultural Field (No. 8) after the tsunami. The white line in the photograph indicates the location of the tide wall before the disaster, its total

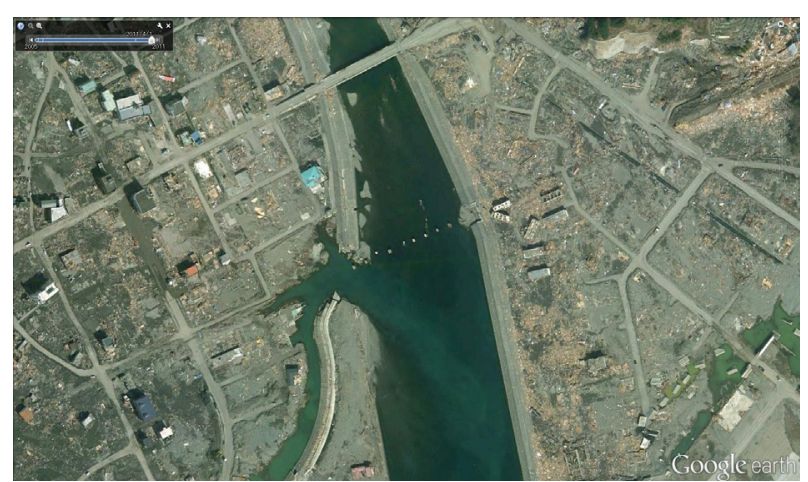

Fig. 9 Aerial photograph of Otsuchi River Bank (No. 6).

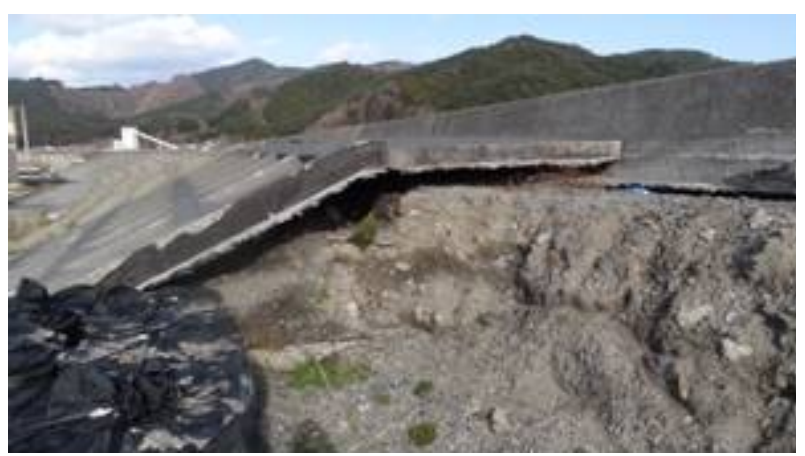

Fig. 10 Damage to tide wall (No. 6).

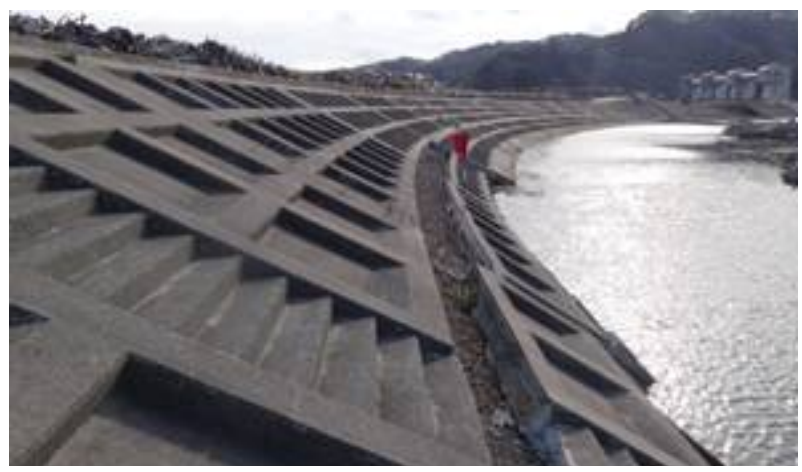

Fig. 11 Slipped off tide wall (No. 6). 


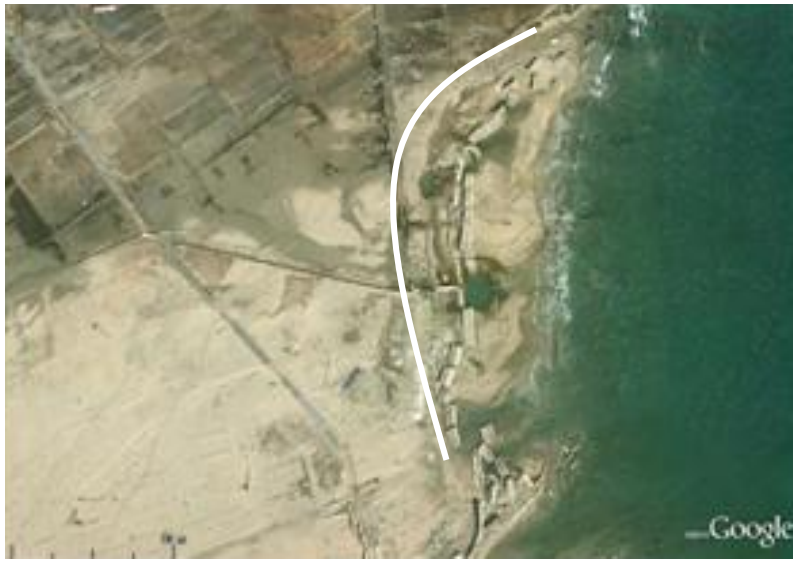

Fig. 12 Aerial photograph of coast at Yoshihama agricultural field (No. 8).

length being around $0.6 \mathrm{~km}$. The wall collapsed toward the sea side along its entire length. The seaward collapse of the main body of the wall can be seen in Fig. 13.

The tide wall on the Koyadori Coast (No. 4) also had the back slope torn off and the infill sand washed out, as shown in Fig. 14. The damage to the front slope of the wall is shown in Fig. 15. Judging from these observations, the backrush is believed to be the reason for the seaward collapse of the tide wall.

In the case of the tide wall on the Mutsugahama Coast (No. 10), the back slope slid down and the infill sand was washed out, as shown in Fig. 16. The damage to the front slope of the same tide wall is shown in Fig. 17. The concrete blocks were bent at around the center in a vertical direction but no displacement of the wall was observed. This situation suggests that a preceding scouring of the infill sand lessened the support of the concrete blocks.

On the Tofugaura Coast (No. 1) located next to the Mutsugahama Coast, a vertical type tide wall had been constructed. The back slope of the wall was lost and part of the infill sand washed out, but the tide wall itself was saved from collapse, as shown in Fig. 18. This wall is known to have been constructed at a different time from the other structures. The foundations of this seawall take root in the bedrock.

As explained above, it can be stated that a vertical type tide wall with back slope protection is liable to

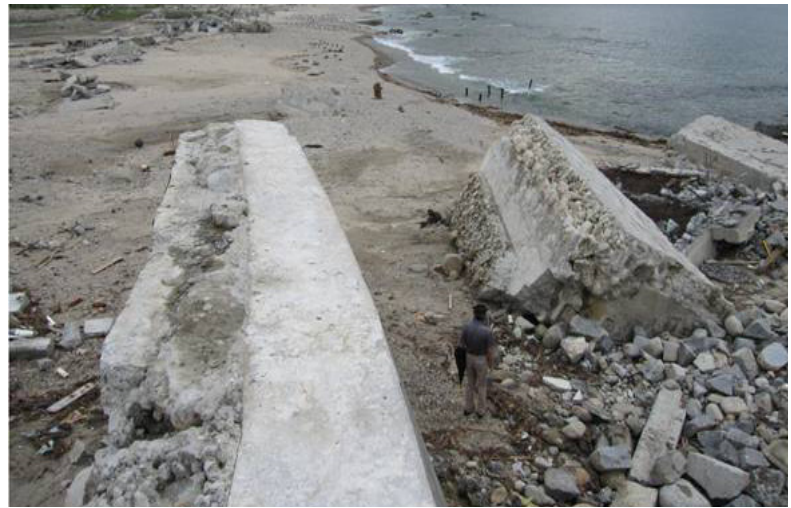

Fig. 13 Damage to tide wall (No. 8).

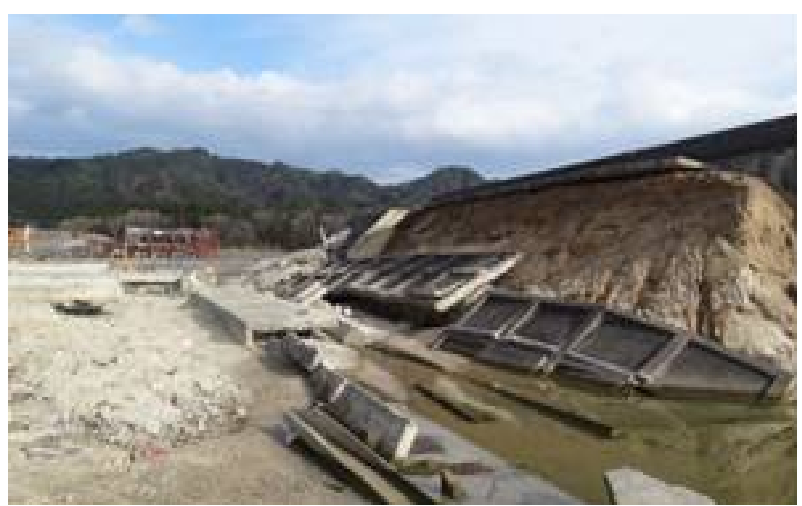

Fig. 14 Slipped off tide wall (No. 4).

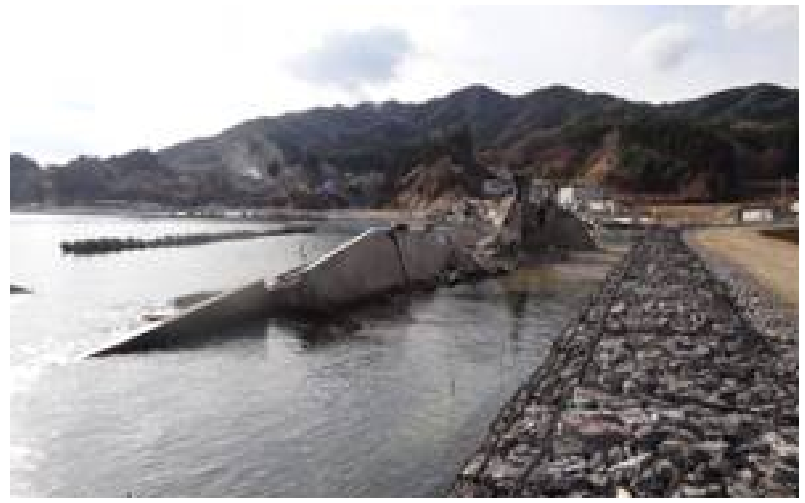

Fig. 15 Damage to tide wall (No. 4).

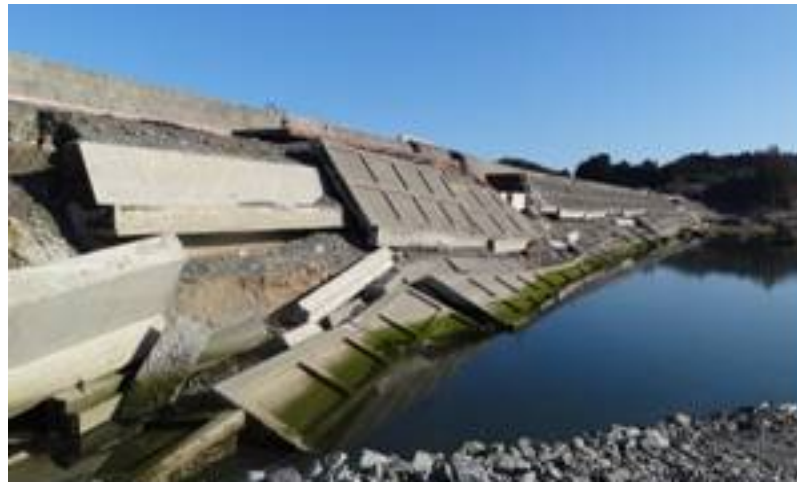

Fig. 16 Slipped off tide wall (No. 10). 


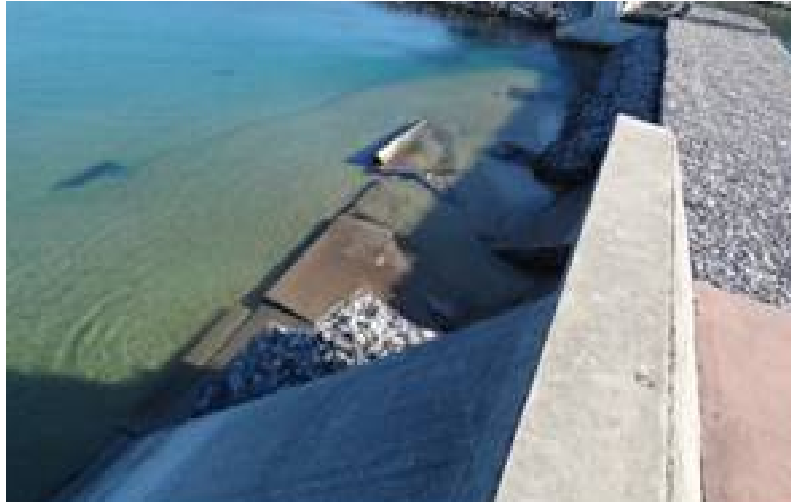

Fig. 17 Damage to tide wall (No. 10).

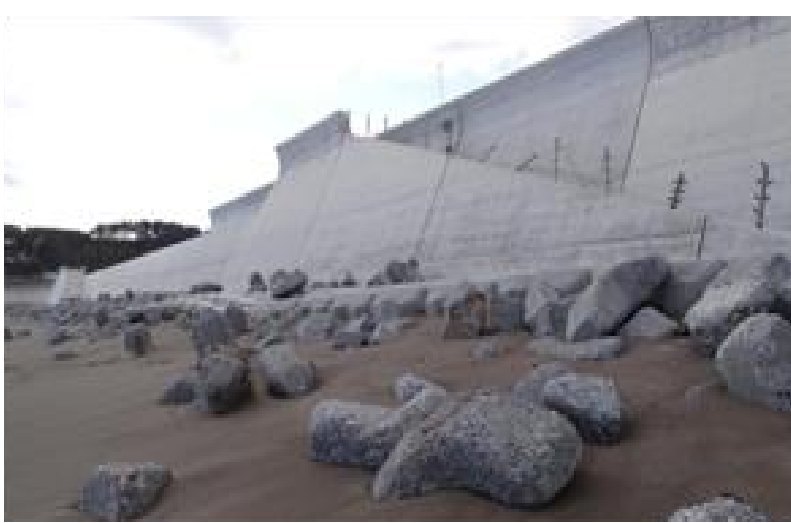

Fig. 18 Tide wall which did not collapse (No. 1).

suffer from damage in the same manner as the sloping type; i.e., scouring of the infill sand of the back slope triggering collapse.

\subsection{Vertical Type Tide Wall without Back Slope Protection}

Fig. 19 shows an aerial photograph of the coast at Yamada Fishery Port (No. 3) after the disaster. In this area, a self-standing tide wall had been constructed, with a total length of $1.3 \mathrm{~km}$. A large part of the tide wall remains nearly unaffected by the tsunami, except for a severely damaged section of length $0.2 \mathrm{~km}$. In the damaged section, some segments fell down from their bases and others had their parapets torn off, with detritus scattered on the land side, as shown in Fig. 20. This indicates that the section collapsed because of spilling waves. It can also be seen in the photograph that there are pools of water on the rear side of the original position of the damaged section, which suggests that overflowing and scouring caused the

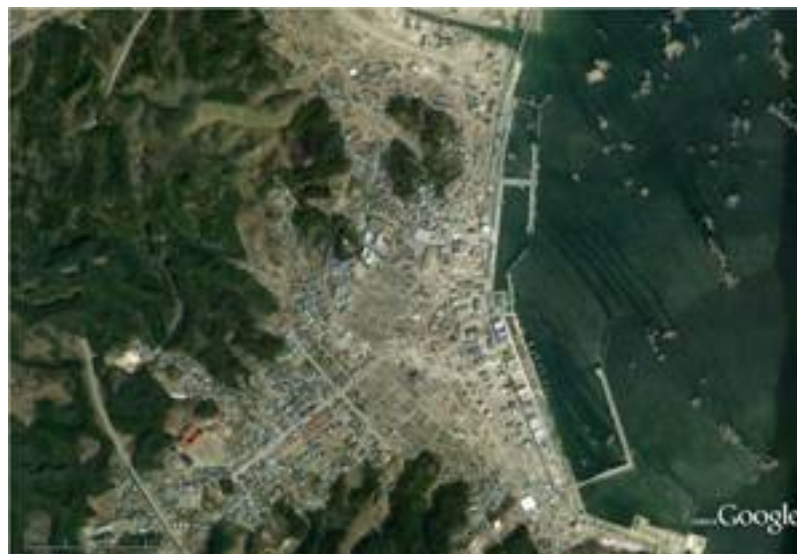

Fig. 19 Aerial photograph of coast at Yamada Fishery Port (No. 3).

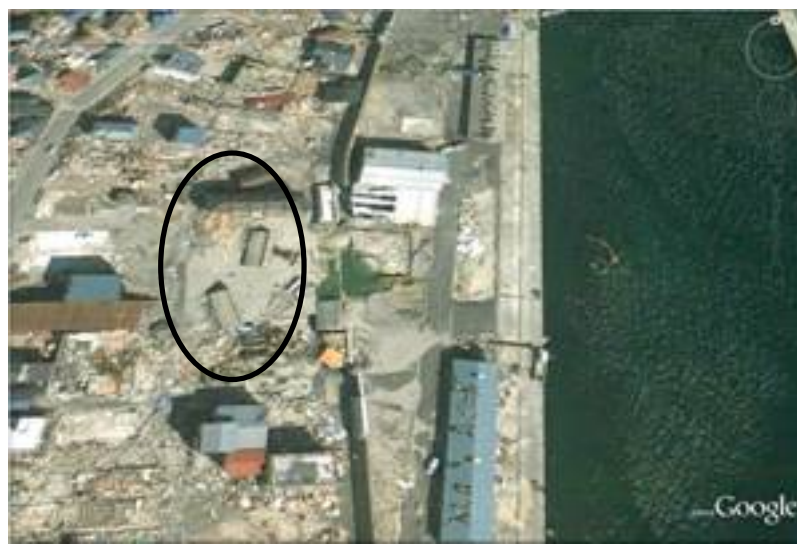

Fig. 20 Damaged tide wall (No. 3).

damage. This idea is supported by the fact that no water was discovered on the rear side of the unaffected section. Because urgent restoration work was in progress when we visited the site and the damaged section was protected by sandbags, we could not confirm the damage that had occurred.

Fig. 21 shows an aerial photograph of the coast at Kojirahama Fishery Port (No. 7) after the disaster. The total length of the tide wall in this area is $0.6 \mathrm{~km}$. The four rectangular blocks in the center of the photograph indicate the location of the damaged section of the wall and the block positions show that the tide wall collapsed or leaned over toward the land side. The damage is shown in Fig. 22. The soil at the rear side of the collapsed tide wall has largely been scoured and pools of water can be observed. Here again, the scouring is believed to be the reason for the collapse. 

Great East Japan Earthquake

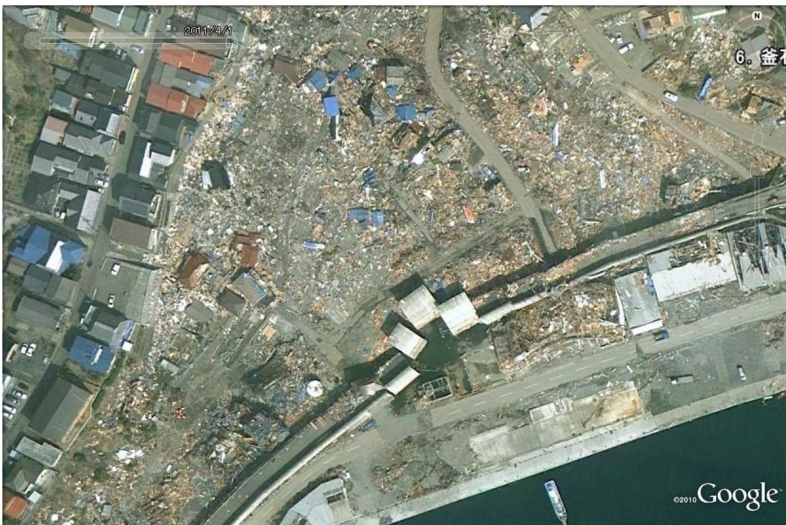

Fig. 21 Aerial photograph of coast at Kojirahama Fishery Port (No. 7).

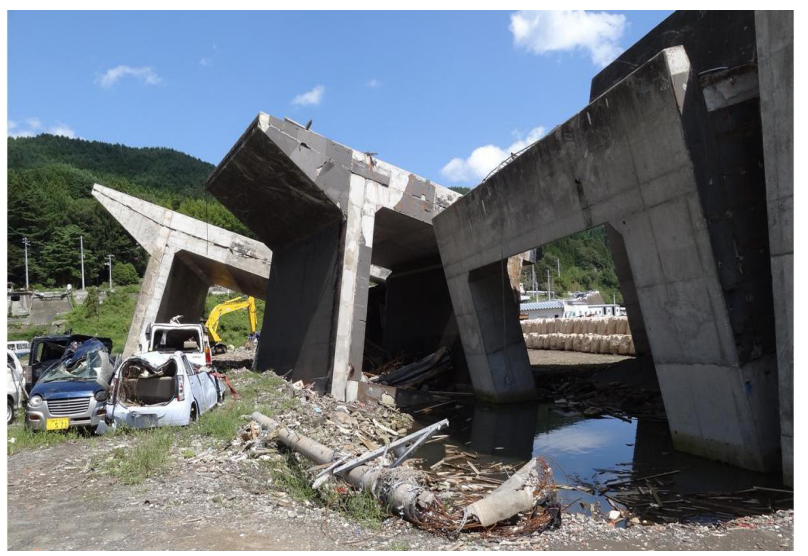

Fig. 22 Damage to tide wall (No. 7).

An aerial photograph of the coast at Kadonohama Fishery Port Coast (No. 9) after the disaster is shown in Fig. 23. The damage in this area is shown in Fig. 24. The damaged section of the tide wall collapsed toward the sea side. The wall at the front of the photograph shows seaward collapse and that at the back indicates landward collapse with drifting toward the sea occurring. Some traces of scouring can be seen on the rear side of the tide wall in other sections saved from collapse, as shown in Fig. 25. The main reason for the scouring is contributed to the overflow by the tsunami, with the situation being the same in the collapsed section.

The characteristics of the damage on vertical type tide walls without back slope protection are therefore considered to be collapse due to spilling waves, or scouring caused by spilling waves.

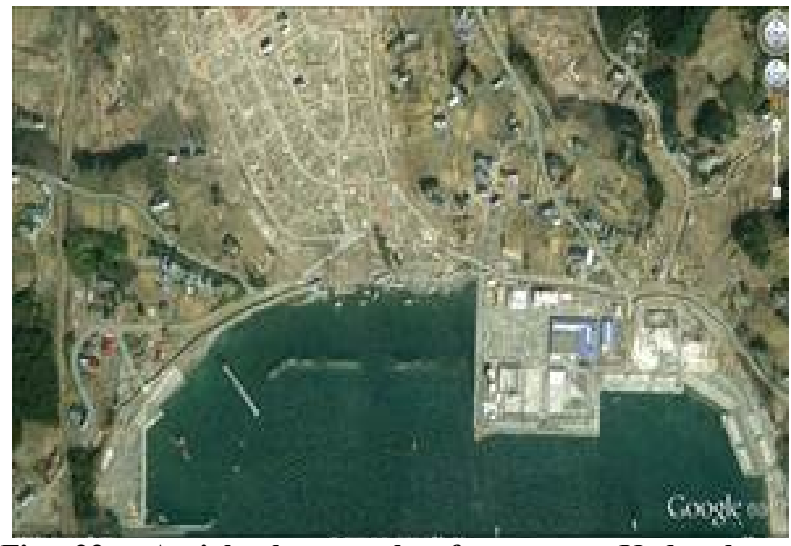

Fig. 23 Aerial photograph of coast at Kadonohama fishery port (No. 9).

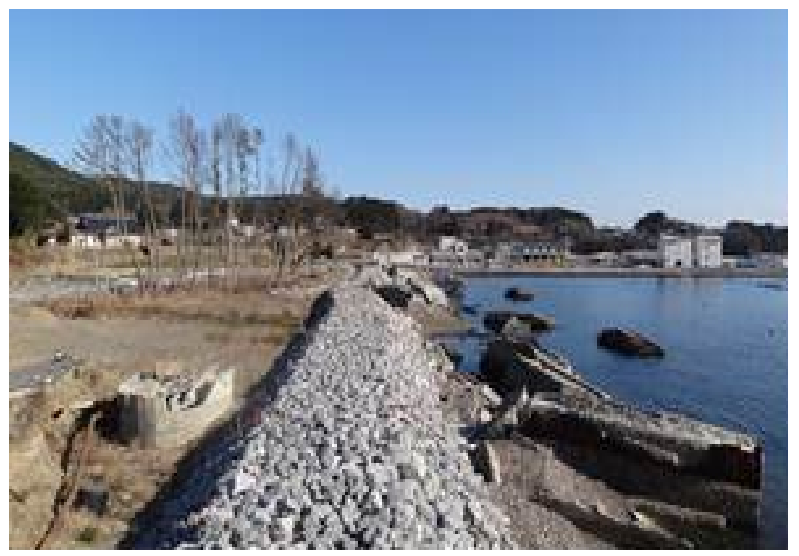

Fig. 24 Damage to tide wall (No. 9).

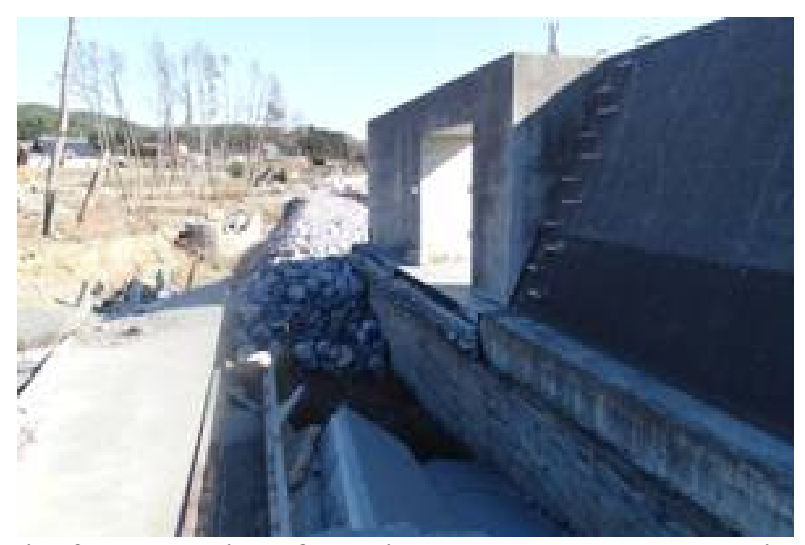

Fig. 25 Rear side of the tide wall at no damage section (No. 9).

\section{Conclusions}

We investigated 13 tide walls damaged by the Great East Japan Earthquake in order to determine the reasons that the damage occurred. Although the scope of our research in this current study is limited, we 
were able to identify the characteristics of the damage to the tide walls we investigated. Our findings can be summarized as follows:

(1) Sloping tide walls collapsed because the back slope protection concrete and infill sand were washed out by the overflow of the tsunami;

(2) Vertical tide walls with back slope protection collapsed in the same manner as sloping tide walls, the infill sand being washed out;

(3) Vertical tide walls without back slope protection collapsed due to spilling waves or scouring of the soil at the rear side of the wall.

It was confirmed that all the tide walls we investigated suffered from overflow by the tsunami. If there had been no overflow, washing out of the soil on the rear side of tide wall and of the infill sand would not have occurred. In fact, the Ohtanabe tide wall in Fudai Village, Iwate Prefecture prevented overflow of the tsunami and thereby protected the lives of the villagers [5]. Furthermore, the tide wall there suffered from almost no damage. This also reinforces our findings that the washing out of the soil and infill sand is the main reasons for the damage to the tide walls. Hence, it is believed that the best measure against tsunamis is to reassess the possible wave heights of future tsunamis and to construct tide walls with appropriate corresponding wall heights.

However, it is not an easy task to forecast the height of possible tsunamis in the future. It has been reported that wooden houses are totally destroyed if the inundation height of a tsunami exceeds $2 \mathrm{~m}$ [6]. If a tide wall can be saved from collapse even after suffering from overflow, it can be expected to resist the tsunamis that follow. The second important measure is, therefore, to protect the tide wall from collapse. Based on our findings, effective methods for achieving this are protection of the tide wall by solidification of the infill sand and soil on the rear side of the wall to an extent that protects them from being washed out, or provision of suitable surface protection for the same purpose.

The formulation of a plan for reinforcing tide walls in anticipation of future tsunamis is an urgent challenge. We hope that our findings can be of some help in achieving this.

\section{Acknowledgments}

This research was conducted as a part of joint research entitled "Detailed investigation of damage by the Great East Japan Earthquake and recommendations for the creation of safe coastal regions" by Iwate Prefecture and the Research Center for Regional Disaster Management of Iwate University Faculty of Engineering. We would like to express our sincere gratitude to the parties concerned.

In addition, we would like to state that all the aerial photographs used in this research paper are from Google Earth.

\section{References}

[1] Advance report on the Great East Japan Earthquake, National Research Institute for Earth Science and Disaster Prevention Web Site, http://www.bosai.go.jp/ saigai/2010/images/20110323_01.pdf (accessed July 15, 2012).

[2] Estimation of Damage to Capital Stock Due to the Great East Japan Earthquake, Homepage of the Development Bank of Japan Web Site, http://www.dbj.jp/ja/topics/dbj_ news/2011/files/0000006633_file1.pdf (accessed July 15, 2012).

[3] Lessons of the Great East Japan Earthquake, Civil Engineering Edition, Overall Picture of Damage to Infrastructure, Nikkei Construction, 2011, pp. 26-29.

[4] Design Manual for Coastal Facilities, Coastal Engineering Committee, Japan Society of Civil Engineering, Nov. 2000.

[5] S. Hanehara, Toward the renaissance of new Tohoku-Iwate, Concrete Journal 50 (1) (2012) 78-81.

[6] N. Shuto, Tsunami Strength and Damage, Tsunami Engineering Technical report, Japan Society of Civil Engineering, 1992, pp. 101-136. 